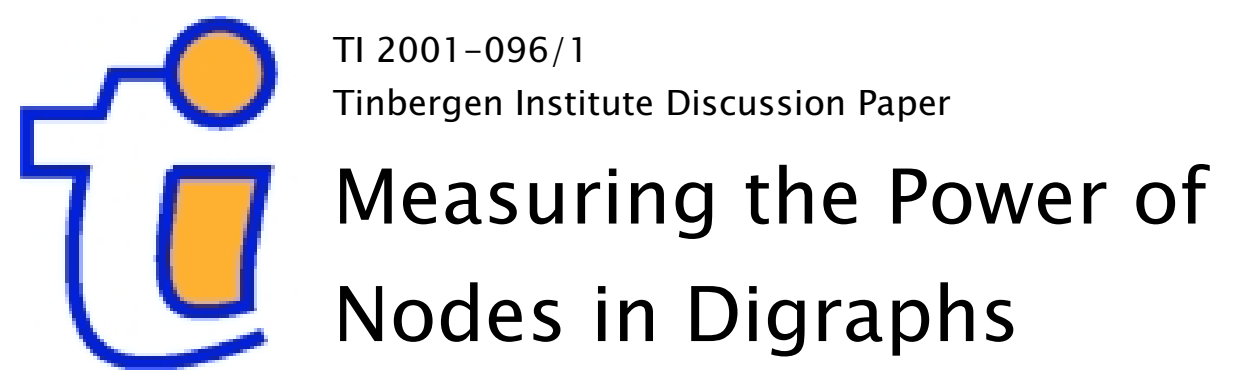

\author{
P. Jean-Jacques Herings ${ }^{7}$ \\ Gerard van der Laan² \\ Dolf Talman
}

1 Department of Economics, International Institute for Infonomics, and METEOR, Universiteit Maastricht, 2 Department of Econometrics, Faculty of Economics and Business Administration, Vrije Universiteit Amsterdam, and Tinbergen Institute, ${ }^{3}$ Department of Econometrics \& Operations Research, and CentER, Tilburg University 
Tinbergen Institute

The Tinbergen Institute is the institute for economic research of the Erasmus Universiteit Rotterdam, Universiteit van Amsterdam and

Vrije Universiteit Amsterdam.

Tinbergen I nstitute Amsterdam

Keizersgracht 482

1017 EG Amsterdam

The Netherlands

Tel.: +31.(0)20.5513500

Fax: $\quad+31 .(0) 20.5513555$

Tinbergen Institute Rotterdam

Burg. Oudlaan 50

3062 PA Rotterdam

The Netherlands

Tel.: $\quad+31 .(0) 10.4088900$

Fax: $\quad+31 .(0) 10.4089031$

Most TI discussion papers can be downloaded at

http://www.tinbergen.nl 


\title{
Measuring the Power of Nodes in Digraphs
}

\author{
P. Jean-Jacques Herings ${ }^{1}$ Gerard van der Laan ${ }^{2}$ Dolf Talman ${ }^{3}$
}

October 3, 2001

${ }^{1}$ P.J.J. Herings, Department of Economics, International Institute for Infonomics, and METEOR, Universiteit Maastricht, P.O. Box 616, 6200 MD Maastricht, The Netherlands, e-mail: p.herings@algec.unimaas.nl

${ }^{2}$ G. van der Laan, Department of Econometrics and Tinbergen Institute, Free University, De Boelelaan 1105, 1081 HV Amsterdam, The Netherlands, e-mail: glaan@econ.vu.nl

${ }^{3}$ A.J.J. Talman, Department of Econometrics \& Operations Research and CentER, Tilburg University, P.O. Box 90153, 5000 LE Tilburg, The Netherlands, e-mail: talman@kub.nl 


\begin{abstract}
Many economic and social situations can be represented by a digraph. Both axiomatic and iterative methods to determine the strength or power of all the nodes in a digraph have been proposed in the literature. We propose a new method, where the power of a node is determined by both the number of its successors, as in axiomatic methods, and the powers of its successors, as in iterative methods. Contrary to other iterative methods, we obtain a full ranking of the nodes for any digraph. The new power function, called the positional power function, can either be determined as the unique solution to a system of equations, or as the limit point of an iterative process. The solution is also explicitly characterized. This characterization enables us to derive a number of interesting properties of the positional power function. Next we consider a number of extensions, like the positional weakness function and the position function.
\end{abstract}

JEL classification: C60, C70, D70

Key words: graph, tournament, power function 


\section{Introduction}

Many economic and social situations can been modelled by means of a digraph. A digraph is an irreflexive directed graph consisting of a finite set $N$ of nodes and a collection $A$ of ordered pairs of these nodes, called arcs or arrows, e.g. see Behzad, Chartrand and Lesniak-Foster [1]. An arc from one node to another node represents a dominance relation of the former node over the latter node. For instance, in a sports competition each node is a player or team and an arc going from node $i$ to node $j$ means that player $i$ has won a play against player $j$, e.g. see Moon and Pullman [19], Rubinstein [22], and Laffont, Laslier and LeBreton [17]. Within an economic perspective, Gilles, Owen and van den Brink [11] and van den Brink [4] model a hierarchical structure by a digraph. Within this framework the nodes represent economic agents and an arc going from node $i$ to node $j$ means that agent $i$ has economic power over agent $j$, e.g. to set trading conditions. In social choice theory the set of nodes represents the available alternatives. Then the problem is that several individuals (voters) may have different, often conflicting, preferences over the available alternatives and that only one alternative can be implemented. The problem to choose the most preferred alternative can be modelled as a digraph by assigning an arc from node $i$ to node $j$ when in a pairwise comparison of the alternatives, alternative $i$ is preferred to $j$ by a majority of the voters, see e.g. David [9], Sen [24], Kano and Sakamoto [14], and the monograph of Laslier [18]. Although usually the individual preferences are transitive, in general the resulting digraph does not reflect a transitive ordering.

A digraph is a tournament when for any two different nodes $i, j \in N$ it holds that either there is an arc from $i$ to $j$ or from $j$ to $i$. Clearly, in a round-robin sports competition the resulting digraph is a tournament when draws are not possible. Also the digraph obtained from the majority voting in the social choice problem is a tournament when for any pair of alternatives the majority voting is decisive, which will be certainly true in case the number of voters is odd and all individual preferences are linear orders. In this paper we consider the class of all digraphs, allowing for non-decisive majority voting in case of social choice theory, an 'open' competition (not requiring that every team meets every other team exactly once), and any type of structure in a (hierarchical) organisation. Moreover, while in social choice situations the decision maker is often only interested in finding an optimal alternative, in a sports competition often not only the winner matters, but one is usually also interested in ranking all the teams. Also in economic (hierarchical) situations it may be important to have a measure of the strength or power of all the nodes. In this paper our aim is to obtain such a full ranking of the nodes in any digraph by measuring for every node the power being exercised directly or indirectly on all other nodes.

To rank the nodes in a digraph, several methods have been proposed in the literature. 
Axiomatic ranking procedures were proposed for example by Sabidussi [23], Nieminen [21], Behzad, Chartrand and Lesniak-Foster [1], Rubinstein [22], Bouyssou [3] , van den Brink [4], and van den Brink and Gilles [6], [7]. An axiomatic procedure yields a unique function $f$ from the collection of all digraphs to the $n$-dimensional real space $\mathbb{R}^{n}$, satisfying certain axioms, where $n$ is the number of nodes. For each digraph $A$, the value $f_{i}(A)$ of the $i$-th component of $f(A) \in \mathbb{R}^{n}$ can be seen as a measure of the power of node $i$ within digraph $A$. From the components of vector $f(A)$ a ranking of the nodes in digraph $A$ is obtained. In the axiomatic approach the problem is to find an independent system of axioms that uniquely determines a function $f$ on the class of all digraphs. Of course, the induced ranking $f(A)$ of the nodes in digraph $A$ depends on the axioms to be satisfied. A well-known axiomatic power function is the score function, in which the power of a node is equal to the number of its successors.

Another class of ranking procedures are the so-called iterative solutions. This type of methods goes back to Wei [26] and Kendall [16]. For a tournament these methods have been discussed extensively in Laslier [18]. The method proposed by Wei [26], called the 'Long path method' by Laslier [18], iterates the score vector. In Moon and Pullman [19], see also Keener [15], it is shown that only under restrictive assumptions on the digraph $A$, this procedure converges to a non-zero vector. Other iterative procedures are the socalled Markov solution proposed by e.g. Daniels [8], see also Laslier [18], and a procedure proposed in Borm, van den Brink and Slikker [2], which is based on the axiomatic power function of van den Brink [4]. All these procedures will be discussed more extensively in Section 2. Here we only state that they all have some serious drawbacks. The Long path method is only well-defined when the digraph satisfies very strong conditions. In particular it must hold that each node must be dominated by at least one other node, excluding for instance digraphs being hierarchies or trees or nodes being isolated. The Markov solution is only well-defined on the class of tournaments, while the procedure of Borm, van den Brink and Slikker [2] gives a unique outcome to any digraph, but has the disadvantage that it only ranks the players in the top cycles. ${ }^{1}$ Although this property is often required in case of the social choice problem of choosing a best alternative, it implies that the procedure cannot be used to obtain a full ranking of all the nodes. Contrary to the axiomatic power functions, the iterative methods measure the global power of nodes in a digraph.

Besides the axiomatic and iterative methods, alternative ranking methods have been proposed by e.g. Slater [25] and Kano and Sakamoto [14]. Both these methods are based on a concept of distance between the digraph and the rankings on the set of nodes. For instance, in Kano and Sakamoto [14], the so-called backward length between the digraph

\footnotetext{
${ }^{1}$ A set $K$ of nodes is a top cycle of digraph $A$ if for any two nodes $i$ and $j$ in $K$, there exists a sequence $i_{1}, \ldots, i_{\ell}$ of nodes such that $i_{1}=i, i_{\ell}=j$ and $\left(i_{k}, i_{k+1}\right) \in A, k=1, \ldots, \ell-1$, and when for any $i \in K$ and $h \notin K$ it holds that $(h, i) \notin A$. Note that there may be multiple top cycles.
} 
and a ranking is minimized on the set of all rankings.

In this paper we propose a new method for measuring the power of the nodes in a digraph. To do so, we first show that all iterative solutions mentioned above can be obtained as a (not necessarily unique) solution of a homogeneous system of linear equations. Then we will formulate an alternative system of linear equations and it will be shown that this system does not suffer from the drawbacks of the iterative procedures mentioned above. In particular, the system has a unique solution for any digraph and therefore the new method is not restricted to a subclass of digraphs. Moreover, the solution vector gives zero power to any dummy node and a positive power to any node that dominates at least one other node, so that it does not only rank the nodes in the top cycles, but also the other nodes. The motivation of the new power function is that the power of a node is determined by both the number of its successors, as in the score measure, and the powers of its successors, as is the case in the iterative methods.

We also introduce a weakness function on the collection of all digraphs, which measures the weakness of the nodes in a digraph, and a position function. The latter function measures the position of any node in a digraph as the difference between its power and its weakness in the digraph and can be seen as the Copeland variant of the new power function.

This paper is organized as follows. In the next section we discuss both the axiomatic solutions and the iterative methods and we show that the solutions generated by these latter methods are eigenvectors of well-chosen systems of equations. In Section 3 we introduce the new power function as the unique solution of a system of equations. Moreover we discuss several properties of the new power function and show that the solution of the system of equations can be seen as the limit point of an iterative process. In Section 4 we propose the weakness function and the Copeland variant of the new power function, while in Section 5 some further generalizations are discussed.

\section{Power Functions on Digraphs}

A directed graph consists of a set of nodes and a set of directed edges or arcs. The set of nodes is denoted by $N$ and consists of a finite number of $n$ elements, indexed by $i=1, \ldots, n$. An arc points from some node $i \in N$ to some node $j \in N$ and is denoted by the ordered pair $(i, j)$. A directed graph on the set $N$ of nodes is denoted by its set $A$ of arcs, i.e. $A \subset N \times N$. If $(i, j) \in A$ we say that node $i$ dominates node $j$. Node $i \in A$ is a dummy node if it does not dominate any other node; node $i \in A$ is a top node if it dominates any node that has at least one predecessor.

A directed graph is called irreflexive if $(i, i) \notin A$ for every $i \in N$. An irreflexive 
directed graph is shortly called a digraph. A digraph $A$ is said to be a tournament if for any two different nodes $i, j \in N$ it holds that either $(i, j) \in A$ or $(j, i) \in A$. A digraph is called to be transitive when for any three nodes $i, j$ and $h$ it holds that $(i, h) \in A$ when both $(i, j) \in A$ and $(j, h) \in A$. Clearly, when $A$ is a transitive tournament then $A$ reflects a linear order on the set of nodes. Finally, digraph $A$ is called empty when $A=\emptyset$ and complete when $A=N \times N \backslash\{(i, i) \mid i \in N\}$. Throughout this paper $\mathcal{A}$ denotes the collection of all digraphs on a given set $N$ of $n$ nodes, i.e. $\mathcal{A}$ is the collection of all irreflexive directed graphs on $N$.

A ranking or power function $f$ on the collection $\mathcal{A}$ of digraphs on $N$ assigns for any $A \in \mathcal{A}$ a real number to every node $i$ in $N$, which can be seen as its strength, as in a sports competition, its power, as in a digraph reflecting a hierarchical structure, or determining its rank, as in a digraph reflecting preferences over a finite set of alternatives. To facilitate the introduction of power functions, we first define for every node $i \in N$ its sets of predecessors and successors in $A$ by

$$
P_{i}^{A}=\{j \in N \mid(j, i) \in A\} \text { and } S_{i}^{A}=\{j \in N \mid(i, j) \in A\}
$$

respectively, i.e. $P_{i}^{A}$ is the set of all nodes by which $i$ is dominated in $N$ and $S_{i}^{A}$ is the set of all nodes in $N$ dominated by $i$. We denote the cardinality of these sets by $p_{i}^{A}$ and $s_{i}^{A}$, respectively, i.e. $p_{i}^{A}=\left|P_{i}^{A}\right|$ and $s_{i}^{A}=\left|S_{i}^{A}\right|, i \in N$. Observe that when $A$ is a tournament we have for all $i$ and all $h \neq i$ that $h$ belongs to either $P_{i}^{A}$ or $S_{i}^{A}$, so that $p_{i}^{A}+s_{i}^{A}=n-1$.

\subsection{Axiomatic power functions}

Two axiomatic power functions on the class $\mathcal{A}$ of all digraphs are, first, the well-known score function, see for instance Behzad, Chartrand and Lesniak-Foster [1], Delver, Monsuur and Storcken [10] or Rubinstein [22], and second, the alternative dominance function, see van den Brink [4] and van den Brink and Gilles [6], [7].

\section{Definition 2.1 (Score and dominance functions)}

(i) The score function is the function $f^{s}: \mathcal{A} \rightarrow \mathbb{R}_{+}^{n}$ given by $f_{i}^{s}(A)=s_{i}^{A}, i \in N, A \in \mathcal{A}$.

(ii) The dominance function is the function $f^{d}: \mathcal{A} \rightarrow \mathbb{R}_{+}^{n}$ given by $f_{i}^{d}(A)=\sum_{j \in S_{i}^{A}} \frac{1}{p_{j}^{A}}$, $i \in$ $N, A \in \mathcal{A}$.

So, for any digraph $A$ the score of a node $i$ is equal to the number of nodes dominated by $i$, i.e. the score is equal to the number of wins. The interpretation of the dominance function is as follows. First, every node being dominated by at least one other node is given one unit of power. Then, for every node this unit is equally distributed over all its predecessors. Consequently, the power of node $i$ is the sum of all the contributions obtained by $i$ from its successors. So, when $i$ dominates node $j$ and $j$ is also dominated by $k-1$ other nodes, 
then the domination of $j$ by $i$ yields a contribution of $\frac{1}{k}$ to the power of $i$. Both the score and dominance function are uniquely defined by a number of properties.

A function $f: \mathcal{A} \rightarrow \mathbb{R}^{n}$ satisfies the dummy node property if for every $A \in \mathcal{A}$ it holds that $f_{i}(A)=0$ if $s_{i}^{A}=0$. Also, $f$ satisfies top node property if for every $A \in \mathcal{A}$ and for every top node $i \in N$, it holds that $f_{i}(A) \geq f_{h}(A)$ for all $h \in N$. The next property says that for any independent partition of a digraph into subdigraphs the power of a node in the digraph is equal to the sum of the powers of the node in each of the subdigraphs. ${ }^{2}$ Formally, a function $f: \mathcal{A} \rightarrow \mathbb{R}^{n}$ satisfies the additivity over independent partitions property if for every $A \in \mathcal{A}$ and for every independent partition $\mathcal{D}=\left\{D_{1}, \ldots, D_{s}\right\}$ of $A$ into $s$ subdigraphs it holds that $f_{i}(A)=\sum_{k=1}^{s} f_{i}\left(D_{k}\right), i \in N$. The next characterization of the score and dominance function has been given in van den Brink [4], see also van den Brink and Gilles [7].

\section{Theorem 2.2}

(i) The score function $f^{s}: \mathcal{A} \rightarrow \mathbb{R}_{+}^{n}$ is the only function $f$ on $\mathcal{A}$ satisfying the dummy node property, the top node property, the additivity over independent partitions property and the normalization rule that the total power is equal to the number of arcs, i.e. for every $A \in \mathcal{A}$ it holds that $\sum_{i \in N} f_{i}(A)=|A|$.

(ii) The dominance function $f^{d}: \mathcal{A} \rightarrow \mathbb{R}_{+}^{n}$ is the only function $f$ on $\mathcal{A}$ satisfying the dummy node property, the top node property, the additivity over independent partitions property and the normalization rule that the total power is equal to the number of dominated nodes, i.e. for every $A \in \mathcal{A}$ it holds that $\sum_{i \in N} f_{i}(A)=\left|\left\{i \in N \mid p_{i}^{A}>0\right\}\right|$.

The theorem implies that the score and dominance function are uniquely determined by the top and dummy node property, the property of additivity over independent partitions and a normalization rule and only differ from each other because of the different normalizations. In Rubinstein [22] a characterization of the ranking by the score function has been given on the subclass of tournaments. In this case the ranking by the score function is the same as the ranking by the well-known Copeland score function, which assigns to every node the difference between the number of successors and the number of predecessors. A characterization of this Copeland score has been given by Bouyssou [3]. For more properties we refer to Behzad, Chartrand and Lesniak-Foster [1] for the score function and to van den Brink and Gilles [6], [7] for the dominance function. In particular, both the score function and dominance function share the symmetry property that $f_{i}(A)=f_{h}(A)$ if both $P_{i}^{A}=P_{h}^{A}$ and $S_{i}^{A}=S_{h}^{A}$.

\footnotetext{
${ }^{2}$ For a digraph $A$, a collection $\mathcal{D}=\left\{D_{1}, \ldots, D_{s}\right\}$ of $s$ digraphs is called a partition of $A$ if $A=\cup_{k=1}^{s} D_{k}$ and $D_{k} \cap D_{\ell}=\emptyset$ for all $k \neq \ell$. A partition $\mathcal{D}=\left\{D_{1}, \ldots, D_{s}\right\}$ is called independent if for any $i$ there is a $D_{k}$ containing all arrows $(j, i)$ in $A$.
} 
We conclude this subsection by mentioning that in van den Brink and Borm [5] the dominance function has been modified to the function $f^{m d}: \mathcal{A} \rightarrow \mathbb{R}_{+}^{n}$ given by $f_{i}^{m d}(A)=$ $\sum_{j \in S_{i}^{A} \cup\{i\}} \frac{1}{1+p_{j}^{A}}, i \in N$, i.e. in the modified dominance function each node distributes its unit of measure equally over itself and all its predecessors. In fact, it is the dominance function applied to the reflexive graph $\widehat{A}$ induced by $A$, i.e. $\widehat{A}=A \cup\{(i, i) \mid i \in N\}$, and thus satisfies that the total power is equal to the number of nodes. However, observe that this modified dominance function does not satisfy the dummy node property on $A$.

\section{$2.2 \quad$ Iterative procedures}

The power assigned to a node according to an axiomatic function can be seen as the power being directly exercised by a node in the digraph. For example, the score power of a node only depends on its number of successors in the digraph, while the (modified) dominance power of a node depends only on the number of predecessors of each of its successors (including the node itself). Power functions obtained by iterative procedures, however, can be seen as global measures because they also take into account how strong these successors are and measure therefore also the indirect power exercised by a node.

First we consider the Long path method or iterated score vector, originated from Wei [26] and Kendall [16], see also Daniels [8], Moon and Pullman [19], Keener [15] and Laslier [18]. For given digraph $A$ this procedure is given by the sequence

$$
x^{t}=T^{A} x^{t-1}, \quad t=1,2, \ldots,
$$

with $x^{0}=e$ with $e_{i}=1$ for $i=1, \ldots, n$ and $T^{A}$ the $n \times n$ adjacency matrix $T^{A}$ of the digraph $A$ with elements $t_{i j}=1$ if $(i, j) \in A$ and $t_{i j}=0$ otherwise. It follows that $x^{1}$ is the score vector $s^{A}, x^{2}$ is the vector giving for any node $i$ the scores of all its successors and so on. This iterative method is not guaranteed to converge to a reasonable solution. For instance, when $A$ is a transitive tournament, then $x^{t}$ converges to the zero vector and so does not give a ranking. Only under severe restrictions the procedure converges to a nonnegative solution. More precisely, let $\widetilde{\mathcal{A}} \subset \mathcal{A}$ be the subset of digraphs such that for all $A \in \widetilde{\mathcal{A}}$ it holds that for any $i$ and $j$ there exists a sequence $i_{1}, i_{2}, \ldots, i_{k}$ such that $i_{1}=i$, $i_{k}=j$ and $\left(i_{h}, i_{h+1}\right) \in A$ for all $h=1, \ldots, k-1$. Such a digraph is called a league and implies that each node is linked to any other node through a sequence of directed edges. In particular it implies that each node is dominated by at least one other node and also dominates at least one other node. We now have the following theorem, see Moon and Pullman [19].

\section{Theorem 2.3}

For any $A \in \widetilde{\mathcal{A}}$, the adjacency matrix $T^{A}$ has a unique positive eigenvalue $\lambda^{A}$. Moreover, 
the iterative system (1) converges to the unique eigenvector (up to normalization) $\operatorname{MP}(A) \in$ $\mathbb{R}^{n}$ of $T^{A}$ with eigenvalue $\lambda^{A}$.

The theorem says that in case of a league the iterative process converges to the unique solution (up to normalization) of the system of equations $x=\lambda^{A} T^{A} x$ with $\lambda^{A}$ the unique positive eigenvalue of $T^{A}$, i.e. the Moon-Pullman power vector $\operatorname{MP}(A)$ satisfies

$$
x_{i}=\lambda^{A} \sum_{j \in S_{i}^{A}} x_{j}, i \in N,
$$

showing that the power of node $i$ is equal to the unique positive eigenvalue of $T^{A}$ times the total power of its successors. The fact that $\operatorname{MP}(A)$ is not defined when $A \notin \widetilde{\mathcal{A}}$ limits the usefulness of this power concept.

The next procedure, called the Markov procedure, has been proposed by Daniels [8] and others, see Laslier [18], and is given by the iterative system

$$
x^{t}=\frac{1}{n-1}\left(T^{A}+S^{A}\right) x^{t-1}, \quad t=1,2, \ldots,
$$

with $x^{0}=e$ and $S^{A}$ the $n \times n$ diagonal matrix with the score $s_{i}^{A}$ of node $i$ on the $i$-th place of the diagonal. When $A$ is a tournament and thus the number of predecessors $p_{i}^{A}$ of node $i$ is equal to $n-1-s_{i}^{A}$ it holds that each column of the matrix $M^{A}=\frac{1}{n-1}\left(T^{A}+S^{A}\right)$ sums up to one and therefore $M^{A}$ is a Markov transition matrix. It is well known from the elementary theory of stochastic processes that for such a Markov matrix the iterative process (3) has a unique limit point $\mathrm{MA}(A)$, being an eigenvector with eigenvalue 1 of the matrix $M^{A}$. So, $\operatorname{MA}(A)$ is a solution of the system $x=M^{A} x$. In case $p_{i}^{A}>0$ it follows that $\mathrm{MA}_{i}(A)$ is given by

$$
\operatorname{MA}_{i}(A)=\frac{1}{p_{i}^{A}} \sum_{j \in S_{i}^{A}} \operatorname{MA}_{j}(A),
$$

i.e. when $p_{i}^{A}>0$ the power of node $i$ is equal to the total power of its successors divided by its number of predecessors. However, the Markov power vector $\operatorname{MA}(A)$ is not defined when $A$ is not a tournament, which limits its usefulness.

The procedure proposed in Borm, van den Brink and Slikker [2] overcomes this drawback. This iterated dominance vector is given by the system

$$
x^{t}=\widehat{T}^{A} x^{t-1}, \quad t=1,2, \ldots,
$$

with $x^{0}=e$ and $\widehat{T}^{A}$ the modified adjacency matrix with elements $\widehat{t}_{i j}^{A}=\frac{1}{p_{j}^{A}+1}$ if $(i, j) \in A$ or when $j=i$, and $\widehat{t}_{i j}=0$ otherwise. So, using equation (5), we obtain for $i=1, \ldots, n$ that $x_{i}^{t}=\sum_{j \in S_{i}^{A} \cup\{i\}} \frac{x_{j}^{t-1}}{1+p_{j}^{A}}$, showing that after the first iteration the vector according to the modified dominance function is obtained. This equation also shows that for any $A \in \mathcal{A}$ each 
column of the matrix $\widehat{T}^{A}$ sums up to one and therefore $\widehat{T}^{A}$ is a Markov transition matrix. Hence, the iterative process (5) has a unique limit point $\operatorname{BBS}(A)$, being an eigenvector with eigenvalue 1 of the matrix $\widehat{T}^{A}$. So, $\operatorname{BBS}(A)$ is a solution of the system $x=\widehat{T}^{A} x$. Again, when $p_{i}^{A}>0$, it follows that $\operatorname{BBS}_{i}(A)$ is given by

$$
\operatorname{BBS}_{i}(A)=\frac{1}{p_{i}^{A}} \sum_{j \in S_{i}^{A}} \frac{p_{i}^{A}+1}{p_{j}^{A}+1} \operatorname{BBS}_{j}(A),
$$

i.e. when $p_{i}^{A}>0$ the power of node $i$ is equal to a weighted sum of the power of its successors divided by its number of predecessors. Notice that this solution differs only from the Markov solution with respect to the weights $\frac{p_{i}^{A}+1}{p_{j}^{A}+1}$ of the powers of the nodes $j \in S_{i}^{A}$. Nevertheless, it turns out that the power vector $\operatorname{BBS}(A)$ is also defined when $A$ is not a tournament.

Still, also this iterative procedure has some drawbacks. First, it should be noticed that for both this process and the Markov process it holds that the procedure converges to an eigenvector with eigenvalue 1 of the corresponding matrix, but that this eigenvector does not need to be the unique (normalized) eigenvector with eigenvalue 1. Second, the solution $\operatorname{BBS}(A)$ only discriminates between the nodes in top cycles (see footnote 1 ). Any node not being in a top cycle gets value equal to zero, even when it dominates many other nodes. For instance, let $A$ be given by $A=\{(1,2)\} \cup\{(2, j) \mid j=3, \ldots, n\}$. Then $A$ has one top cycle, only containing node 1 , and so $\mathrm{BBS}_{1}(A)=n$ and $\mathrm{BBS}_{j}(A)=0$ for $j=2, \ldots, n$. $^{3}$ Given the fact that node 1 only dominates node 2 and node 2 dominates all other nodes this outcome is not very satisfactory. Further, the solution does not satisfy the dummy node property. In particular, an isolated node (being a top cycle on its own) gets value 1 . So, the outcome $\operatorname{BBS}(A)$ is useful when there is only a need to select a best alternative in a social choice situation, the winner in a sports competition, or the strongest agent in an organizational structure, but cannot be used to get a full ranking of all nodes.

\section{The Positional Power of Nodes}

The solutions to the iterative procedures can be seen as global power measures of the nodes in the digraph. The systems of equations (2), (4) and (6) show that in all iterative solutions, the power of a node depends in one way or another on the powers of its successors, which on their turn depend on the power of their successors, and so on. So, according to these measures, the power of a node depends on the powers of all other nodes within the

\footnotetext{
${ }^{3}$ Observe that the sum of the components of $\operatorname{BBS}(A)$ equals $n$, because $x_{i}^{0}=1$ for all $i$. This also reflects the fact that in the modified dominance function each node distributes one unit of measure over its predecessors, including the node itself.
} 
digraph, whereas the axiomatic power functions measure only the local power of a node. The power function to be introduced in this section combines the direct local feature of an axiomatic power function and the indirect global power measure of the iterated power functions.

The idea of the new function is that the power of a node is determined by both the number of its successors, as in the score measure, and the powers of its successors, as in the Long path method. To be more precise, when node $i$ dominates node $j$, then node $i$ gets a fraction $\frac{1}{n}$ of the power of node $j$ plus a fixed amount $\frac{1}{n}$. Potentially the power of node $j$ plus a fixed amount of 1 is distributed equally over all $n$ nodes, but only the nodes that dominate $j$ get their part. So, the power $x_{i}$ of the node $i$ in digraph $A, A \in \mathcal{A}$, is defined by

$$
x_{i}=\sum_{j \in S_{i}^{A}}\left(\frac{1}{n} x_{j}+\frac{1}{n}\right), i \in N .
$$

The next theorem shows that for every digraph $A \in \mathcal{A}$ this system of equations has a unique nonnegative solution. Notice that the system is not homogeneous, contrary to the systems corresponding to the iterative procedures discussed in Section 2.

\section{Theorem 3.1}

For every digraph $A$, the system of equations (7) has a unique solution. Moreover, all components of this solution are nonnegative.

\section{Proof}

Rewriting the system of equations (7) in matrix notation we obtain

$$
\left(I-\frac{1}{n} T^{A}\right) x=\frac{1}{n} s^{A},
$$

with $I$ the identity matrix of appropriate dimension, $T^{A}$ the adjacency matrix of $A$ and $s^{A}$ the score vector of $A$. Let $b_{i j}$ be the $(i, j)$-th element of the matrix $B^{A}=I-\frac{1}{n} T^{A}$. Since $b_{i i}=1$ for all $i$ and $b_{i j} \leq 0$ for all $i \neq j$, according to Hawkins and Simon [12] the inverse of $B^{A}$ exists and is nonnegative iff there exists a nonnegative vector $y \in \mathbb{R}^{n}$ such that each component of $z=B^{A} y$ is positive. Take $y=e$, the vector with all components equal to one. Then, $z_{i}=\sum_{j=1}^{n} b_{i j}=1-\sum_{\left\{j \neq i \mid t_{i j}=1\right\}} \frac{1}{n} \geq 1-\frac{n-1}{n}>0$, where $t_{i j}$ is the $(i, j)$-th element of the matrix $T^{A}$. Hence, $z$ is strictly positive and therefore the inverse of $B^{A}$ exists and all elements of the inverse are nonnegative. Since also the vector $s^{A}$ is nonnegative, it follows that system (8) has a unique nonnegative solution $x^{A}$ given by

$$
x^{A}=\frac{1}{n}\left(I-\frac{1}{n} T^{A}\right)^{-1} s^{A} .
$$


The solution of the system of equations (7) measures the power of the position of the nodes within the digraph $A$. The positional power function is now defined as follows.

Definition 3.2 The positional power function is the function $f^{p}: \mathcal{A} \rightarrow \mathbb{R}^{n}$ which assigns to every $A \in \mathcal{A}$ the vector $f^{p}(A)=\frac{1}{n}\left(I-\frac{1}{n} T^{A}\right)^{-1} s^{A}$.

According to Theorem 3.1 the positional power function is well-defined and assigns a nonnegative power vector $f^{p}(A)$ to any digraph $A \in \mathcal{A}$. This overcomes the drawback of the Moon-Pullman solution $\operatorname{MP}(A)$, which is restricted to the subclass $\widetilde{\mathcal{A}}$ of leagues, and the drawback of the Markov solution $\operatorname{MA}(A)$, which is restricted to tournaments.

The positional power function satisfies several nice properties. First, $f^{p}$ satisfies the dummy node property, i.e. $f_{i}^{p}(A)=0$ if $S_{i}^{A}=\emptyset$. Moreover, since $x^{A}$ is a nonnegative vector it also follows immediately from the system of equations (7) that $x_{i}^{A}>0$ when $S_{i}^{A} \neq \emptyset$, implying that the positional power function assigns zero power to a node if and only if it is a dummy node. So, the power function $f^{p}$ also overcomes the drawback of the iterative dominance solution $\operatorname{BBS}(A)$, which assigns zero power to any node not in a top cycle on the one hand and positive power to an isolated node on the other hand. From the system of equations (7) it follows that $f_{i}^{p}(A) \geq f_{j}^{p}(A)$ if $S_{j}^{A} \subset S_{i}^{A}$ with strict inequality when $S_{j}^{A}$ is a proper subset of $S_{i}^{A}$. This 'monotonicity' property implies that $f^{p}$ satisfies the top node property and is symmetric (in fact, any two nodes having the same set of successors have the same power). When $A$ is a tournament there is a unique top cycle and we have that $S_{j}^{A} \subset S_{i}^{A} \backslash\{j\}$ and thus $f_{i}^{p}(A)>f_{j}^{p}(A)$ for any $i$ in the top cycle and any $j$ not in the top cycle. So, when applying the positional power function to select a best alternative in a social choice problem, the function selects an alternative from the top cycle. Summarizing, we have the following properties.

\section{Corollary 3.3}

The positional power function $f^{p}: \mathcal{A} \rightarrow \mathbb{R}_{+}^{n}$ satisfies for any $A \in \mathcal{A}$ the following properties.

- For every node $i \in N$ it holds that $f_{i}^{p}(A)>0$ if and only if $S_{i}^{A} \neq \emptyset$.

- For every pair of nodes $i, j \in N$ it holds that $f_{j}^{p}(A) \geq f_{i}^{p}(A)$ if $S_{i}^{A} \subset S_{j}^{A}$ with equality only when $S_{i}^{A}=S_{j}^{A}$.

- When $A$ is a tournament, $f_{i}^{p}(A)>f_{j}^{p}(A)$ for any $i$ in the top cycle and any $j$ not in the top cycle.

Before discussing more properties of $f^{p}$ we derive the following lemma about the inverse matrix $\left(I-\frac{1}{n} T^{A}\right)^{-1}$. In the sequel we denote this matrix by $V^{A}$, with $(i, j)$-th element equal to $v_{i j}$. 


\section{Lemma 3.4}

For any pair $i, j=1, \ldots, n$, the elements $v_{i j}$ of $V^{A}$ have the following properties.

(i) $v_{i i}=1+\sum_{h \in P_{i}^{A}} v_{i h} / n$ and $v_{i j}=\sum_{h \in P_{j}^{A}} v_{i h} / n$ for $j \neq i$.

(ii) $\sum_{j=1}^{n}\left(n-s_{j}^{A}\right) v_{i j}=n$.

(iii) $1 \leq v_{i i} \leq 2 n /(n+1)$ and $0 \leq v_{i j} \leq n /(n+1)$ for $j \neq i$.

(iv) $v_{i j}=0$ for $j \neq i\left(v_{i i}=1\right)$ if and only if there does not exists an ordered path of arcs from node $i$ to node $j$ (node $i$ ).

\section{Proof}

Since $V^{A}\left(I-\frac{1}{n} T^{A}\right)=I$, we find by rearranging terms that $V^{A}=I+\frac{1}{n} V^{A} T^{A}$. Recalling that the $(h, j)$-th element $t_{h j}$ of the matrix $T^{A}$ is equal to 1 if $h \in P_{j}^{A}$ and 0 otherwise, we obtain property (i). Postmultiplying both sides of the equality $V^{A}\left(I-\frac{1}{n} T^{A}\right)=I$ by the vector $e$, we obtain for any $i=1, \ldots, n$

$$
\sum_{j=1}^{n} v_{i j}=1+\sum_{j=1}^{n} \sum_{h \in P_{j}^{A}} v_{i h} / n=1+\sum_{h=1}^{n} \sum_{j \in S_{h}^{A}} v_{i h} / n=1+\sum_{h=1}^{n} s_{h}^{A} v_{i h} / n,
$$

which, by rearranging terms, yields property (ii). From the proof of Theorem 3.1 we already know that $v_{i j} \geq 0$ for all $j \neq i$ and thus from property (i) it follows that $v_{i i} \geq 1$ for all $i=1, \ldots, n$. Since $s_{h}^{A} \leq n-1$ for all $h$, it follows from property (ii) that

$$
\sum_{h=1}^{n} v_{i h} \leq \sum_{h=1}^{n}\left(n-s_{h}^{A}\right) v_{i h}=n
$$

Hence, for $j \neq i$, from property (i) we obtain

$$
v_{i j}=\sum_{h \in P_{j}^{A}} v_{i h} / n \leq \sum_{h=1}^{n} v_{i h} / n-v_{i j} / n \leq 1-v_{i j} / n,
$$

because $j \notin P_{j}^{A}$. This shows that $v_{i j} \leq n /(n+1)$ for $j \neq i$. Similarly, we obtain

$$
v_{i i} \leq 1+\sum_{h=1}^{n} v_{i h} / n-v_{i i} / n \leq 2-v_{i i} / n
$$

To prove property (iv), notice that since all $t_{i j} \geq 0, t_{i i}=0$ and $\sum_{j \neq i} \frac{1}{n} t_{i j}<1$, we have that the inverse matrix $V^{A}$ can be written as

$$
V^{A}=I+\sum_{k=1}^{\infty} \frac{1}{n^{k}}\left(T^{A}\right)^{k}
$$

with $\left(T^{A}\right)^{k}$ the product of $k$ matrices $T^{A}$. Clearly, $\left(\left(T^{A}\right)^{k}\right)_{i j}>0$ if and only if there exists at least one ordered path of adjacent arcs of length $k$ from node $i$ to node $j$. This implies 
that if there is no path at all from node $i$ to node $j$ we must have that $v_{i i}=1$ and $v_{i j}=0$ when $j \neq i$, and conversely.

Q.E.D.

The next result follows easily from the previous lemma.

\section{Lemma 3.5}

For any $A \in \mathcal{A}$ it holds that $f^{p}(A)=\left(V^{A}-I\right)$.

\section{Proof}

From equation (9) it follows that $f^{p}(A)=\frac{1}{n} V^{A} s^{A}$ and thus

$$
f_{i}^{p}(A)=\sum_{j=1}^{n} s_{j}^{A} v_{i j} / n, i=1, \ldots, n .
$$

From property (ii) of Lemma 3.4 we obtain

$$
f_{i}^{p}(A)=\sum_{j=1}^{n} v_{i j}-1, i=1, \ldots, n
$$

which proves the lemma.

Q.E.D.

Since equation (10) shows that $V^{A}-I=\sum_{k=1}^{\infty} \frac{1}{n^{k}}\left(T^{A}\right)^{k}$ it follows from Lemma 3.5 that $f^{p}(A)=\left(\sum_{k=1}^{\infty} \frac{1}{n^{k}}\left(T^{A}\right)^{k}\right) e$ and thus that for any starting vector $x^{0}$, the power vector $f^{p}(A)$ is the limit point of the iterative process

$$
x^{t}=\frac{1}{n} T^{A}\left(x^{t-1}+e\right), t=1,2, \ldots
$$

Taking as starting vector $x^{0}=0$, we obtain that $x^{1}=\frac{1}{n} s^{A}$, i.e. the first iteration gives a (normalized) score vector, which corresponds to the first iteration of the Long path method. However, any next iteration differs because of the fixed term $\frac{1}{n} T^{A} e$, giving to a node $i$ a fraction $\frac{1}{n}$ of the current power of its successors plus the fixed amount $\frac{1}{n}$ for each of its its successors. For any $i, j=1, \ldots, n$, the nonnegative number $\frac{1}{n^{k}}\left(\left(T^{A}\right)^{k}\right)_{i j}, k \in \mathbb{N}$, is precisely the contribution of node $j$ to the power of node $i$ over all ordered paths of length $k$ in $A$ leading from node $i$ to node $j$. Remark that a path may contain several cycles and contain a cycle more than once. Adding up all these contributions over $k \in \mathbb{N}$ yields the total contribution $v_{i j}$ of node $j$ to the power of node $i \neq j$ (respectively $v_{i i}-1$ when $i=j$ ). Adding up all these total contributions over $j$ yields the positional power of node $i$ in the $\operatorname{digraph} A$. Recall from property (iv) of Lemma 3.4 that $v_{i j}\left(v_{i i}-1\right.$ if $\left.i=j\right)$ is positive if and only if there exists at least one ordered path from node $i$ to node $j$.

The next lemma shows that the positional power function is increasing in $A$ and that adding an arc from $h$ to $k$ increases the power of node $h$ more than the power of any other node. 


\section{Lemma 3.6}

Let $A$ and $A^{\prime}$ be two digraphs such that $A^{\prime}=A \cup\{(h, k)\}$ for some $(h, k)$ not in $A$. Then the following properties hold for $f^{p}(A)$ and $f^{p}\left(A^{\prime}\right)$.

(i) For any $i=1, \ldots, n, f_{i}^{p}\left(A^{\prime}\right) \geq f_{i}^{p}(A)$.

(ii) $f_{h}^{p}\left(A^{\prime}\right)-f_{h}^{p}(A)>\max _{i \in N \backslash\{h\}} f_{i}^{p}\left(A^{\prime}\right)-f_{i}^{p}(A)$.

(iii) For every $i \neq h, f_{i}^{p}\left(A^{\prime}\right)=f_{i}^{p}(A)$ if and only if there is no ordered path of any length in $A$ from node $i$ to node $h$.

\section{Proof}

Let $E^{h, k}=T^{A^{\prime}}-T^{A}$, so element $(i, j)$ of the matrix $E^{h, k}$ is equal to 1 for $(i, j)=(h, k)$ and equal to zero otherwise. Let $\Delta x^{A}=x^{A^{\prime}}-x^{A}$. Since $\left(I-\frac{1}{n} T^{A}\right) x^{A}=\frac{s^{A}}{n},\left(I-\frac{1}{n} T^{A^{\prime}}\right) x^{A^{\prime}}=\frac{s^{A^{\prime}}}{n}$ and $s^{A^{\prime}}=s^{A}+e^{h}$, where $e^{h}$ is the $h$-th unit vector in $\mathbb{R}^{n}$, by subtracting the first equality from the second one we obtain that

$$
\left(I-\frac{1}{n} T^{A^{\prime}}\right) \Delta x^{A}-\frac{1}{n} E^{h, k} x^{A}=\frac{e^{h}}{n}
$$

and so

$$
\left(I-\frac{1}{n} T^{A^{\prime}}\right) \Delta x^{A}=\frac{1}{n} E^{h, k} x^{A}+\frac{e^{h}}{n}=\left(\frac{x_{k}^{A}}{n}+\frac{1}{n}\right) e^{h} .
$$

Therefore,

$$
\Delta x^{A}=c V^{A^{\prime}} e^{h}
$$

where $c=\frac{1}{n}\left(x_{k}^{A}+1\right)$. From the proof of Theorem 3.1 it follows that $V^{A^{\prime}}$ is a nonnegative matrix. Since $c$ is a positive number and $e^{h}$ is a nonnegative vector, it follows that $\Delta x^{A}$ is a (nonzero) nonnegative vector, which proves property (i). From $\Delta x^{A}=c V^{A^{\prime}} e^{h}$ it follows that for $i=1, \ldots, n$,

$$
\Delta x_{i}^{A}=c v_{i h}^{\prime}
$$

where $v_{i h}^{\prime}$ is the $(i, h)$-th element of the matrix $V^{A^{\prime}}$. Applying property (iii) of Lemma 3.4 to $V^{A^{\prime}}$ we obtain for $i \neq h$

$$
\Delta x_{h}^{A}=c v_{h h}^{\prime} \geq c>c v_{i h}^{\prime}=\Delta x_{i}^{A},
$$

which proves property (ii). Finally, when $A$ does not contain an ordered path from node $i \neq h$ to node $h$, then also $A^{\prime}$ does not contain such a path. So, according to property (iv) of Lemma 3.4 applied to $V^{A^{\prime}}$ we have that $v_{i h}^{\prime}=0$ and thus $\Delta x_{i}^{A}=0$, which proves property (iii).

Q.E.D.

Finally, let us consider the total positional power being assigned to the nodes in a digraph $A$. The total positional power of a digraph $A, \sum_{i=1}^{n} f_{i}^{p}(A)$, can be interpreted as a measure of the total amount of power being exercised by the nodes of the digraph $A$. 


\section{Lemma 3.7}

The following properties hold for the total power assigned by the positional power function.

(i) $\sum_{i=1}^{n} f_{i}^{p}(A)=e^{\top} V^{A} e-n$.

(ii) $|A| / n \leq \sum_{i=1}^{n} f_{i}^{p}(A) \leq|A|$.

(iii) $\sum_{i=1}^{n} f_{i}^{p}(A)=0$ if $A$ is the empty digraph, while $\sum_{i=1}^{n} f_{i}^{p}(A)=n(n-1)$ in case $A$ is complete.

\section{Proof}

Clearly, from Lemma 3.5 it follows that

$$
\sum_{i=1}^{n} f_{i}^{p}(A)=e^{\top}\left(V^{A}-I\right) e=e^{\top} V^{A} e-n,
$$

which shows property (i). By the system of equations (7),

$$
\sum_{i=1}^{n} f_{i}^{p}(A)=\sum_{i=1}^{n} \sum_{j \in S_{i}^{A}}\left(\frac{1}{n} f_{j}^{p}(A)+\frac{1}{n}\right)=\sum_{j=1}^{n} \frac{p_{j}^{A}}{n} f_{j}^{p}(A)+|A| / n,
$$

which implies immediately that $\sum_{i=1}^{n} f_{i}^{p}(A) \geq|A| / n$. Rearranging terms gives $\sum_{i=1}^{n}(n-$ $\left.p_{i}^{A}\right) f_{i}^{p}(A)=|A|$, from which it follows that $\sum_{i=1}^{n} f_{i}^{p}(A) \leq|A|$, thereby showing property (ii). Since $p_{j}^{A}=0$ and $|A|=0$ in case of the empty digraph and $p_{j}^{A}=n-1$ and $|A|=n(n-1)$ in case of the complete digraph, $\sum_{i=1}^{n} f_{i}^{p}(A)=0$ if $A$ is the empty digraph, while in case $A$ is complete

$$
\sum_{i=1}^{n} f_{i}^{p}(A)=n-1+\sum_{i=1}^{n} \frac{n-1}{n} f_{i}^{p}(A)
$$

and thus $\sum_{i=1}^{n} f_{i}^{p}(A)=n(n-1)$, which proves property (iii).

Q.E.D.

The Lemma's 3.6 and 3.7 show that the total positional power of the nodes in a graph is strictly increasing in $A$ and lies between $|A| / n$ and $|A|$. It should be noticed that, although the total positional power is increasing in $A$, it is not like the score function (strictly) increasing in the number of arcs. Observe that the total positional power is equal to the number of arcs when $A$ is either the empty or the complete digraph. In both cases the positional power vector is equal to the score vector.

\section{The Positional Weakness and the Net Power of a Node}

While the score function $f^{s}$ assigns to a node its number of successors (wins), $f_{i}^{s}(A)=s_{i}^{A}$, the Copeland score function, denoted by $f^{C}$, is given by its number of successors (wins) 
minus its number of predecessors (losses), i.e. $f_{i}^{C}(A)=s_{i}^{A}-p_{i}^{A}, i=1, \ldots, n$. The function which assigns to every node $i$ the number of predecessors, $p_{i}^{A}$, can be seen as a measure of the (local) weakness of node $i$ within the digraph $A$, while the score function is a measure for the (local) strength or power of node $i$ within $A$. The Copeland score of a node is therefore the difference between the power and the weakness and measures the local position of the node within the digraph, without taking into account how strong the successors or how weak the predecessors are. For the positional power function defined in the previous section we can also define its counterpart, which we call the positional weakness function.

Definition 4.1 The positional weakness function is the function $f^{w}: \mathcal{A} \rightarrow \mathbb{R}_{+}^{n}$ which assigns to every $A \in \mathcal{A}$ the vector $f^{w}(A)=y^{A}$, where $y^{A}$ is the solution of the system of equations given by

$$
y_{i}=\sum_{j \in P_{i}^{A}}\left(\frac{1}{n} y_{j}+\frac{1}{n}\right), i \in N .
$$

Notice that the positional weakness vector of a digraph is equal to the positional power vector of its transpose, where for a digraph $A \in \mathcal{A}$ the transpose $A^{\top}$ is obtained from $A$ by taking the reverse of all arcs, i.e.

$$
A^{\top}=\{(i, j) \in N \times N \mid(j, i) \in A\} .
$$

The positional weakness function measures the weakness of the position of the nodes within a digraph, taking into account the weakness of the predecessors. Similar to the positional power function, the positional weakness function is well defined. Obviously, results analogous to the ones of Section 3 can be shown for the positional weakness function, for instance that it assigns to every digraph a nonnegative vector, $f_{i}^{w}(A)>0$ if and only if $P_{i}^{A} \neq \emptyset$, $f_{j}^{w}(A) \geq f_{i}^{w}(A)$ if $P_{i}^{A} \subset P_{j}^{A}$, and the positional weakness vector increases in $A$. The difference between the positional power and the positional weakness of a node measures net power of that node within the digraph.

Definition 4.2 The net power function is the function $P: \mathcal{A} \rightarrow \mathbb{R}^{n}$ which assigns to every $A \in \mathcal{A}$ the vector $P(A)=f^{p}(A)-f^{w}(A)$.

A positive net power of a node means that the node exercises more power than it suffers from weakness, while a negative net power means the opposite. The net power function can also be used to rank the nodes. The node with the highest net power is the strongest node in the digraph, while the node with the lowest net power is the weakest node. The next lemma shows that the sum of all net powers of the nodes is always equal to zero.

\section{Lemma 4.3}

For any digraph $A \in \mathcal{A}$ it holds that $\sum_{i=1}^{n} P_{i}(A)=0$. 


\section{Proof}

Since $T^{A^{\top}}=\left(T^{A}\right)^{\top}$, we have that $V^{A^{\top}}=\left(I-\frac{1}{n} T^{A^{\top}}\right)^{-1}=\left(V^{A}\right)^{\top}$. Since $f^{w}(A)=f^{p}\left(A^{\top}\right)$ and $f^{p}(A)=\left(V^{A}-I\right) e$, we obtain $f^{w}(A)=\left(\left(V^{A}\right)^{\top}-I\right) e$. Hence, $P(A)=f^{p}(A)-f^{w}(A)=$ $\left(V^{A}-\left(V^{A}\right)^{\top}\right) e$, and thus $\sum_{i=1}^{n} P_{i}(A)=e^{\top}\left(V^{A}-\left(V^{A}\right)^{\top}\right) e=0$.

Q.E.D.

The property that the total power in any digraph is equal to the total power in its transpose digraph also holds for the score function, but not for the dominance function. According to the score function the total power (score) is equal to the number of arcs and hence equal in both digraphs. However, the total power in the dominance function is equal to the total number of dominated nodes, which does not need to be equal in $A$ and its transpose $A^{\top}$.

\section{Some Further Extensions}

In this section we consider some extensions of the positional power function. First, we can extend the concept of positional power function to a class of power functions by generalizing the system of equations (7) to the system

$$
x_{i}=\sum_{j \in S_{i}^{A}}\left(\frac{1}{k} x_{j}+c\right), i \in N,
$$

for any given $c>0$ and $k \geq n-1$. For $k<n-1$ the inverse of the matrix $I-\frac{1}{k} T$ may not exist or contain negative elements, so that the solution $x^{A}$ cannot be guaranteed to be unique or nonnegative. When $k=n-1$ the inverse matrix exists and is nonnegative for any $A$, except when $A$ is complete, in which case the matrix is singular. ${ }^{5}$ Hence, it is reasonable to restrict the values of $k$ to $k>n-1$. In these cases Theorem 3.1 applies and the solution of the corresponding system of equations (7) is given by

$$
\widetilde{x}^{A}=c\left(I-\frac{1}{k} T^{A}\right)^{-1} s^{A} .
$$

From this equation it can be seen that the number $c$ only determines the absolute value of the solution, but not the relative powers or the ranking of the nodes. When $A$ is not empty and thus at least one node has positive power, the constant $c$ can be used to normalize the sum of the powers to one, which in some applications may be useful. This is achieved by setting $c$ equal to $c^{A}$, where

$$
c^{A}=\left(e^{\top}\left(I-\frac{1}{k} T^{A}\right)^{-1} s^{A}\right)^{-1} .
$$

\footnotetext{
${ }^{4}$ For the modified dominance function the total power is always equal to the number of nodes and thus independent of the arcs in the digraph.

${ }^{5}$ Although one could argue that in a complete digraph the power of every node is infinite.
} 
For example, the normalized positional power function can be used to determine whether a collection of nonempty digraphs is balanced, see Herings, van der Laan, and Talman (2000). Given the normalized positional power function, denoted by $f^{n, p}$, a collection $\left\{A^{i} \in \mathcal{A} \mid i \in I_{k}\right\}$ of (nonempty) digraphs is said to be balanced if the system

$$
\sum_{i=1}^{k} \lambda_{i} f^{n, p}\left(A^{i}\right)=\frac{1}{n} e
$$

has a nonnegative solution. Balancedness of digraphs can be used to give sufficient conditions for the nonemptiness of the core as solution concept for a nontransferable utility game in graph structure.

From $\sum_{i=1}^{n} \widetilde{x}_{i}^{A}=c|A|+\sum_{i=1}^{n} \frac{p_{i}^{A}}{k} \widetilde{x}_{i}^{A}$ it follows that when $A$ is the complete digraph, and thus $p^{A}=(n-1) e$, the sum of the powers is equal to

$$
\sum_{i=1}^{n} \widetilde{x}_{i}^{A}=c \frac{k n(n-1)}{k-n+1}
$$

which goes to infinity when $k$ goes to $n-1$, reflecting that the inverse matrix does not exist in case $k=n-1$ and $A$ is complete. On the other hand, $\frac{k n(n-1)}{k-n+1}$ converges to $|A|=n(n-1)$ when $k$ goes to infinity. The latter reflects the fact that up to normalization the generalized positional power function converges to the score function when $k$ goes to infinity. So, the score function can be considered to be the limit case of the class of power functions defined in the system of equations (14).

Above we assumed that every arc in a digraph has equal weight. In case arcs have different weights it is easy to adapt the positional power function accordingly. If $w_{i j}$ is the (nonnegative) weight of arc $(i, j)$ in digraph $A$, then we may consider the system

$$
x_{i}=\sum_{j \in S_{i}^{A}}\left(\frac{w_{i j}}{k} x_{j}+\frac{w_{i j}}{k}\right), i \in N
$$

with $k$ equal to $\left(\max _{i, j} w_{i j}\right) n$. Notice that we allow weights to be larger than one. This might occur for example in a competition where the teams (nodes) play against each other more than once or when a win yields extra points, e.g. one point in case of a draw and three points for a win and zero points for a loss.

\section{References}

[1] M. Behzad, G. Chartrand and L. Lesniak-Foster, Graphs and Digraphs, (Wadsworth, Belmont, USA, 1979).

[2] P. Borm, R. van den Brink and M. Slikker, An iterative procedure for evaluating digraph competitions, Research Memorandum FEW 788, Tilburg University, Tilburg, The Netherlands, 2000. 
[3] D. Bouyssou, Ranking methods based on valued preference relations: a characterization of the net flow method, European Journal of Operational Research 60, (1992) 61-67.

[4] R. van den Brink, Relational Power in Hierarchical Organizations, Ph-D Dissertation, Tilburg University, Tilburg, The Netherlands, 1994.

[5] R. van den Brink and P. Borm, Digraph competitions and cooperative games, Tinbergen Discussion Paper 95-142, Tinbergen Institute, Amsterdam-Rotterdam, The Netherlands, 1995.

[6] R. van den Brink and R.P. Gilles, Ranking the nodes in directed and weighted directed graphs, mimeo, Tilburg University, Tilburg, The Netherlands, 1999.

[7] R. van den Brink and R.P. Gilles, Measuring domination in directed networks, Social Networks 22, (2000) 141-157.

[8] H.E. Daniels, Round robin tournament scores, Biometrika 56 (1969) 295-299.

[9] H.A. David, The Method of Paired Comparisons (Griffin, London, UK, 1963).

[10] R. Delver, H. Monsuur and A.J.A. Storcken, Ordering pairwise comparison structures, Theory and Decision 31, (1991) 75-94.

[11] R.P. Gilles, G. Owen and J.R. van den Brink, Games with permission structures: the conjunctive approach, International Journal of Game Theory 20, (1992) 277-293.

[12] D. Hawkins and H.A. Simon, Note: some conditions on macro-economic stability, Econometrica 17, (1949) 245-248.

[13] P.J.J. Herings, G. van der Laan, and A.J.J. Talman, Cooperative games in graph structure, CentER Discussion paper 2000-52, CentER, Tilburg University, Tilburg, The Netherlands, 2000.

[14] M. Kano and A. Sakamoto, Ranking the vertices of a paired comparison digraph, SIAM Journal on Algebraic and Discrete Methods 6, (1985) 79-92.

[15] J.P. Keener, The Perron-Frobenius theorem and the ranking of football teams, SIAM Review 35, (1993) 80-93.

[16] M.G. Kendall, Further contributions tothe theory of paired comparisons, Biometrics 11, (1955) 43-62. 
[17] G. Laffond, J.F. Laslier and M. LeBreton, The bipartisan set of a tournament game, Games and Economic Behavior 5, (1994) 182-201.

[18] J.F. Laslier, Tournament Solutions and Majority Voting (Springer, Berlin, 1997).

[19] J.W. Moon and N.J. Pullman, On generalized tournament matrices, SIAM Review 12 (1970) 384-399.

[20] H. Moulin, Choosing from a tournament, Social Choice and Welfare 7, (1986) 271-291.

[21] U.J. Nieminen, On the centrality in a directed graph, Social Science Research 2, (1973) 371-378.

[22] A. Rubinstein, Ranking the participants in a tournament, SIAM Journal of Applied Mathematics 38, (1980) 108-111.

[23] G. Sabidussi, The centrality index of a graph, Psychometrika 31, (1966) 581-603.

[24] A.K. Sen, Collective Choice and Social Welfare, (North-Holland, Amsterdam, The Netherlands, 1979).

[25] P. Slater, Inconsistencies in a schedule of paired comparisons, Biometrika 48 (1961), 303-312.

[26] T. Wei, The Algebraic Foundation of Ranking Theory, Ph.D. Thesis, Cambridge University, Cambridge, 1952. 